\title{
CLRg Property for Coupled Fixed Point Theorems in Fuzzy Metric Spaces
}

\author{
M. Alamgir Khan and Sumitra
}

\begin{abstract}
In this paper, we introduce the notions of E.A. property and common limit in range of $\mathrm{g}$ (CLRg) property for coupled mappings and prove a common coupled fixed point theorems without exploiting the notion of continuity, completeness of the whole space or any of its range spaces. Our theorems generalize the result of [5] and [10-14]. We also find an affirmative answer in fuzzy metric space to the problem of Rhoades [2]. Illustrative examples supporting our results have also been cited.
\end{abstract}

Index Terms - Coupled fixed point, weakly compatible maps, E.A. property and (CLRg) property.

\section{INTRODUCTION}

As fuzzy mathematics is the hottest area of research now-a-days and new concepts are emerging very rapidly in this field and consequently it has opened a new venue for many mathematicians. See [1], [2], [4], [5], [6], [7], [8] etc.

Recently, Bhaskar and Lakshmikantham [3] introduced the concepts of coupled fixed points and mixed monotone property and illustrated these results by proving the existence and uniqueness of the solution for a periodic boundary value problem. Later on these results were extended and generalized by Sedghi et al. [7] , Fang [4] and Xin-Qi Hu [5] etc.

In the study of common fixed points of compatible mappings we often require assumption on completeness of the space or continuity of mappings involved besides some contractive condition but the study of fixed points of non comapatible mappings can be extend to the class of non expansive or Lipschitz type mapping pairs even without assuming the continuity of the mappings involved or completeness of the space. Aamri and El Moutawakil [1] generalized the concepts of non comapatibility by defining the notion of (E.A) property and proved common fixed point theorems under strict contractive condition. Although E.A property is generalization of the concept of non compatible maps yet it requires either completeness of the whole space or any of the range space or continuity of maps. But on contrary, the new notion of $\operatorname{CLR}(\mathrm{g})$ property recently given by Sintunavarat and Kuman [8]does not impose such conditions. The importance of CLRg property ensures that one does not require the closeness of range subspaces.

The intent of this paper is to establish the concept of E.A. property and (CLRg) property for coupled mappings and an affirmative answer of question raised by Rhoades[2] whether,

Manuscript received May 19, 2012; revised June, 13, 2012.

M. A. Khan is with the Department of Mathematics, University of Kurdistan, Erbil (e-mail: alam_alam3333@yahoo.com).

Sumitra is with the Department of Mathematics, Jazan University, Saudi Arabia(e-mail: mathsqueen_d@yahoo.com). by using the concept of non comapatibility or its generalized notion, can we find equally interesting results in fuzzy metric space also ?

So, our improvement in this paper is four fold as

1) Relaxed continuity of maps completely

2) Completeness of the whole space or any of its range space removed.

3) Minimal type contractive condition used.

4) The condition $\lim _{t \rightarrow \infty} M(x, y, t)=1$ is not used.

\section{DEFINITIONS AND PRELIMINARIES}

\section{A. Definition 2.1[9].}

A binary operation $*:[0,1] \times[0,1] \rightarrow[0,1]$ is a continuous t-norm if $([0,1], *)$ is a topological abelian monoid with unit 1 s.t. $a * b \leq c * d$ whenever $a \leq c$ and $b \leq d, \forall a, b, c, d \in$ $[0,1]$.

\section{B. Definition 2.2[9].}

The 3-tuple $(X, M, *)$ is called a fuzzy metric space if $X$ is an arbitrary set, $*$ is a continuous t-norm and $\mathrm{M}$ is a fuzzy set on $X^{2} \times[0, \infty)$ satisfying the following conditions:

(FM-1) $M(x, y, 0)>0$,

(FM-2) $M(x, y, \mathrm{t})=1$ iff $x=y$,

(FM-3) $M(x, y, t)=M(y, x, t)$,

(FM-4) $M(x, y, t) * M(y, z, s) \leq M(x, z, t+s)$,

$(\mathrm{FM}-5) M(x, y,):.(0, \infty) \rightarrow[0,1]$ is continuous for all $x, y$, $z \in X$ and $s, t>0$.

\section{Definition 2.3[6].}

An element $(x, y) \in X \times X$ is called a

(i) coupled fixed point of the mapping $f: X \times X \rightarrow X$ if $f(x$, $y)=x, f(y, x)=y$.

(ii) coupled coincidence point of the mappings $f: X \times X \rightarrow$ $X$ and $g: X \rightarrow X$ if $f(x, y)=g(x), \quad f(y, x)=g(y)$.

(iii) common coupled fixed point of the mappings $f: X \times X$ $\rightarrow X$ and $g: X \rightarrow X$ if

$$
x=f(x, y)=g(x), \quad y=f(y, x)=g(y) .
$$

D. Definition 2.4[5].

An element $x \in X$ is called a common fixed point of the mappings $\mathrm{f}: X \times X \rightarrow X$ and

$\mathrm{g}: X \rightarrow X$ if $\quad x=f(x, x)=g(x)$.

\section{E. Definition 2.5 .}

Let $A, B: X \times X \rightarrow X$ and $S, T: X \rightarrow X$ be four mappings. Then, the pair of maps $(B, S)$ and $(A, T)$ are said to have Common Coupled Coincidence Point if there exist $a, b$ in $X$ such that

$B(a, b)=S(a)=T(a)=A(a, b)$ and $B(b, a)=S(b)=T(b)=$ $A(b, a)$. 


\section{F. Definition 2.6.}

The mappings $f: X \times X \rightarrow X$ and $g: X \rightarrow X$ are called weakly compatible maps if

$f(x, y)=g(x), f(y, x)=g(y)$ implies $g f(x, y)=f(g x, g y), g f(y$, $x)=f(g y, g x)$, for all $x, y$ in $X$.

Now, we fuzzify the newly defined concepts of E.A Property introduced by Aamri and Moutawakil [1] and (CLRg) property given by Sintunavarat and Kuman [7] for coupled maps as follows

\section{G. Definition 2.7}

Let $(X, M, *)$ be a FM space. Two maps $f: X \times X \rightarrow X$ and $\mathrm{g}$ : $X \rightarrow X$ are said to satisfy E.A. property if there exist sequences $\left\{x_{n}\right\}$ and $\left\{y_{n}\right\}$ in $X$ such that

$\lim _{n \rightarrow \infty}\left(f\left(x_{n}, y_{n}\right)=\lim _{n \rightarrow \infty} g x_{n}=x \quad\right.$ and $\lim _{n \rightarrow \infty}\left(f\left(y_{n}, x_{n}\right)=\lim _{n \rightarrow \infty} g y_{n}=y\right.$, for some $\mathrm{x}, \mathrm{y}$ in $\mathrm{X}$.

\section{H. Definition 2.8}

Let $(X, M, *)$ be a FM space .Two maps $f: X \times X \rightarrow X$ and $\mathrm{g}$ : $X \rightarrow X$ are said to satisfy (CLRg) property if there exist sequences $\left\{x_{n}\right\}$ and $\left\{y_{n}\right\}$ in $X$ such that

$\lim _{n \rightarrow \infty} M\left(f\left(x_{n}, y_{n}\right), g x_{n}, t\right)=x=g(p)$

$\lim _{n \rightarrow \infty}\left(f\left(y_{n}, x_{n}\right), g y_{n}, t\right)=y=g(q)$, for some $\mathrm{p}, \mathrm{q}$ in $\mathrm{X}$.

1) Example 2.1.

Let $(X, M, *)$ be a fuzzy metric space, * being a continuous norm with $X=R$. Define

$M(x, y, t)=\frac{t}{t+|x-y|}$ for all $x, y$ in $X$ and $t>0$. Define mappings $f: X \times X \rightarrow X$ and $g: X \rightarrow X$ by

$f(x, y)=x-y$ and $g(x)=2 x$ for all $x, y$ in $X$ and consider the sequences $x_{n}=\left\{\frac{1}{n}\right\}$ and $y_{n}=\left\{-\frac{1}{n}\right\}$, then

$$
f\left(x_{n}, y_{n}\right)=\frac{2}{n}, g\left(x_{n}\right)=\frac{2}{n} \Rightarrow \lim _{n \rightarrow \infty} M\left(f\left(x_{n}, y_{n}\right), g\left(x_{n}\right), t\right) \rightarrow 1=g\left(\frac{1}{2}\right)
$$

$f\left(y_{n}, x_{n}\right)=-\frac{2}{n}, g\left(y_{n}\right)=-\frac{2}{n} \Rightarrow \lim _{n \rightarrow \infty} M\left(f\left(y_{n}, x_{n}\right), g\left(y_{n}\right), t\right) \rightarrow 1=g\left(\frac{1}{2}\right)$

therefore, $\mathrm{f}$ and $\mathrm{g}$ satisfy both the. properties E.A and (CLRg )

2) Remark 2.1.

From above examples we can say that E. A does not imply CLR $(g)$. In the next example, we show that the maps satisfying $\operatorname{CLR}(\mathrm{g})$ property need not be continuous.

3) Example 2.2

Let $(X, M, *)$ be a fuzzy metric space, * being a continuous norm with $X=[0, \infty)$. Define $M(x, y, t)=\frac{t}{t+|x-y|}$ for all $x, y$ in $X$ and $t>0$.Define mappings $f: X \times X \rightarrow X$ and $g: X \rightarrow X$ as follows

and

$$
f(x, y)=\left\{\begin{aligned}
x+y & \text { if } x \in[0,1), y \in X \\
\frac{x+y}{2} & \text { if } x \in[1, \infty), y \in X
\end{aligned}\right.
$$

$$
g(x)=\left\{\begin{array}{cl}
1+x & \text { if } x \in[0,1) \\
\frac{x}{2} & \text { if } x \in[1, \infty)
\end{array}\right.
$$

We consider the sequences $\left\{x_{n}\right\}=\left\{\frac{1}{n}\right\}$ and $\left\{y_{n}\right\}=\{1+$ $\left.\frac{1}{n}\right\}$.Then, the maps $\mathrm{f}$ and $\mathrm{g}$ satisfy (CLRg) property but the maps are not continuous.

\section{MAIN RESULTS}

Now, we prove the following result for quadruple maps.

1) Theorem 3.1.

Let $(X, M, *)$ be a Fuzzy Metric Space, * being continuous t-norm with $a * b \geq a b, \forall a, b \in[0,1]$. Let $A, B: X \times X \rightarrow$ $X$ and $S, T: X \rightarrow X$ be four mappings satisfying following conditions:

1) The pairs $(A, S)$ and $(B, T)$ satisfy $\operatorname{CLR}(g)$ property

2) $\quad M(A(x, y), B(u, v), k t) \geq \phi\{M(S x, T u, t) \times M(A(x, y), S x, t) \times$ $M(B(u, v), T u, t)\}$

$$
\forall x, y, u, v \in X, k \in(0,1) \text { and } \phi:[0,1] \rightarrow[0,1]
$$

such that $\phi(t)>t$ for $0<t<1$. Then $(\mathrm{A}, \mathrm{S})$ and $(B, T)$ have point of coincidence. Moreover if the pairs $(A, S)$ and $(B, T)$ are weakly compatible, then there exists unique $x$ in $X$ such that $A(x, x)=T(x)=B(x, x)=S(x)=x$.

2) Proof

Since the pairs $(A, S)$ and $(B, T)$ satisfy CLRg property, there exist sequences $\left\{x_{n}\right\},\left\{y_{n}\right\}\left\{x_{n}^{\prime}\right\}$ and $\left\{y_{n}^{\prime}\right\}$ in $X$ such that $\lim _{n \rightarrow \infty} A\left(x_{n}, y_{n}\right)=\lim _{n \rightarrow \infty} S\left(x_{n}\right)=S a$,

$\lim _{n \rightarrow \infty} A\left(y_{n}, x_{n}\right)=\lim _{n \rightarrow \infty} S\left(y_{n}\right)=S b$ and $\lim _{n \rightarrow \infty} B\left(x_{n}^{\prime}, y_{n}^{\prime}\right)$ $=\lim _{n \rightarrow \infty} \mathrm{T}\left(x_{n}^{\prime}\right)=\mathrm{Ta}^{\prime}, \lim _{n \rightarrow \infty} B\left(y_{n}^{\prime}, x_{n}^{\prime}\right)=\lim _{n \rightarrow \infty} \mathrm{T}\left(y_{n}^{\prime}\right)=$ $\mathrm{Tb}^{\prime}$, for some $a, b, a^{\prime}, b^{\prime}$ in $X$

Step 1: We now show that the pairs $(A, S)$ and $(B, T)$ have common coupled coincidence point. We first show that $S a=$ $T a^{\prime}$.Using (3.2), we have,

$$
\begin{aligned}
& M\left(A\left(x_{n}, y_{n}\right), B\left(x_{n}, y_{n}\right), k t\right) \geq \phi\left\{M\left(S x_{n}, T x_{n}, t\right) \times M\left(A\left(x_{n}, y_{n}\right), S x_{n}, t\right) \times\right. \\
& \left.M\left(B\left(x_{n}, y_{n}\right), T x_{n}, t\right)\right\}
\end{aligned}
$$

Taking $n \rightarrow \infty$, we get

$$
\begin{aligned}
& M\left(S a, T a^{\prime}, k t\right) \geq \phi\left\{M\left(S a, T a^{\prime}, t\right) * 1 * 1\right\} \geq \phi\left\{M\left(S a, T a^{\prime}, t\right)\right. \\
& \geq\left\{M\left(S a, T a^{\prime}, t\right)\right.
\end{aligned}
$$

$$
\text { i.e } \quad M\left(S a, T a^{\prime}, k t\right) \geq M\left(S a, T a^{\prime}, t\right) \Rightarrow S a=T a^{\prime} \text {, }
$$
similarly we can have $S b=T b^{\prime}$.

Also,

$$
\begin{aligned}
& M\left(A\left(y_{n}, x_{n}\right), B\left(x_{n}^{\prime}, y_{n}^{\prime}\right), k t\right) \geq \phi\left\{M\left(S y_{n}, T x_{n}^{\prime}, t\right) \times M\left(A\left(y_{n}, x_{n}\right), S y_{n}, t\right) \times\right. \\
& \left.M\left(B\left(x_{n}^{\prime}, y_{n}^{\prime}\right), T x_{n}^{\prime}, t\right)\right\}
\end{aligned}
$$

i.e $M\left(S b, T a^{\prime}, k t\right) \geq M\left(S b, T a^{\prime}, t\right) \Rightarrow S b=T a^{\prime}$

Hence $S b=T a^{\prime}=S a=T b^{\prime}$. Now, for all $t>0$, using condition (3.2), we have 
$M\left(A\left(x_{n}, y_{n}\right), B\left(a^{\prime}, b^{\prime}\right), k t\right) \geq \phi\left\{M\left(S x_{n}, T a^{\prime}, t\right) \times M\left(A\left(x_{n}, y_{n}\right), S x_{n}, t\right) \times\right.$ $\left.M\left(B\left(a^{\prime}, b^{\prime}\right), T a^{\prime}, t\right)\right\}$

Taking $n \rightarrow \infty$, we get,

$$
\begin{aligned}
& M\left(S a, B\left(a^{\prime}, b^{\prime}\right), k t\right) \geq M\left(S a, B\left(a^{\prime}, b^{\prime}\right), t\right) \Rightarrow \mathrm{Sa} \\
& =\mathrm{B}\left(a^{\prime}, b^{\prime}\right) .
\end{aligned}
$$

Similarly, we can get that $S b=B\left(b^{\prime}, a^{\prime}\right)$. In a similar fashion, we can have $T a^{\prime}=A(a, b)$ and $T b^{\prime}=A(b, a)$.

Thus, $B\left(a^{\prime}, b^{\prime}\right)=S a=T a^{\prime}=A(a, b)$ and $B\left(b^{\prime}, a^{\prime}\right)=S b=$ $T b^{\prime}=A(b, a)$. Thus the pairs $(A, S)$ and $(B, T)$ have coincidence points.

Let $S a=A(a, b)=B\left(a^{\prime}, b^{\prime}\right)=T a^{\prime}=x$ and $S b=A(b, a)$ $=B\left(b^{\prime}, a^{\prime}\right)=T b^{\prime}=y$. Since $(A, S)$ and $(B, T)$ are weakly compatible, so

$$
S x=S A(a, b)=A(S a, S b)=A(x, y)
$$

and

$$
\begin{gathered}
S y=S A(b, a)=A(S b, S a)=A(y, x) . \\
T x=T B\left(a^{\prime}, b^{\prime}\right)=B\left(T a^{\prime}, T b^{\prime}\right)=B(x, y)
\end{gathered}
$$

and

$$
T y=T B\left(b^{\prime}, a^{\prime}\right)=B\left(T b^{\prime}, T a^{\prime}\right)=B(y, x) .
$$

Step 2: We next show that $x=y$. From (3.2),

$$
\begin{aligned}
& M(x, y, k t)=M\left(A(a, b), B\left(a^{\prime}, b^{\prime}\right), k t\right) \\
& \geq \phi\left\{M\left(S a, T a^{\prime}, t\right) \times M(A(a, b), T a, t) \times M\left(B\left(a^{\prime}, b^{\prime}\right), T d, t\right)\right\}=1
\end{aligned}
$$

Thus, $x=y$.

Step 3: Now, we prove that $S x=T x$, using (3.2) again

$$
M(S x, T x, k t)=M(A(x, y), B(y, x), k t) \geq \phi\{M(S x, T y, t) \times
$$$$
M(A(x, y), S x, t) \times M(B(y, x) T y, t)\}
$$

$$
\begin{aligned}
& \phi\{M(S x, T x, t) * M(A(x, y), S x, t) * \\
& M(B(y, x) T y, t)\}
\end{aligned}
$$

i.e $M(S x, T x, k t) \geq M(S x, T y, t) \Rightarrow S x=T x=T y$.

Step 4: Lastly, we prove that $S x=x$

$$
\begin{aligned}
& M(S x, x, k t)=M(S x, y, k t)=M(A(x, y), B(x, y), k t) \\
& \geq \phi\{M(S x, T x, t) * M(A(x, y), S x, t) * M(B(x, y), \\
& T x, t)\}
\end{aligned}
$$

Hence $x=S x=T x=A(x, x)=B(x, x)$. This shows that $A, B, S, T$ have a common fixed point and uniqueness of $x$ follows easily from (3.2).

Next, we give an example in support of theorem 3.1

3) Example 3.1.

Let $X=[-2,2], a * b=a b$ for all $a, b \in[0,1]$ and

$$
M(x, y, t)=\left\{\begin{array}{l}
\frac{t}{t+|x-y|}, t \neq 0 \\
0, t=0
\end{array}\right\} .
$$

Then

$(X, M, *)$ is a Fuzzy Metric space. Define the mappings $A, B: X \times X \rightarrow X$ and $S, T: X \rightarrow X$ as follows

$$
A(x, y)=\left\{\begin{array}{l}
x+y, x \in[0,2], y \in X \\
1, \text { otherwise }
\end{array}\right\}
$$

and

$$
\begin{gathered}
B(x, y)=\left\{\begin{array}{l}
x-y, x \in[0,2], y \in X \\
2, \text { otherwise }
\end{array}\right\} \\
S(x)=\left\{\begin{array}{l}
x, x \in[0,2], y \in X \\
1, \text { otherwise }
\end{array}\right\}
\end{gathered}
$$

and

$$
T(x)=\left\{\left\{\begin{array}{l}
x, x \in[0,2], y \in X \\
2, \text { otherwise }
\end{array}\right\}\right\}
$$

Consider the sequences,

$$
\begin{aligned}
& x_{n}=\left\{\frac{1}{n}\right\}, y_{n}=\left\{-\frac{1}{n}\right\}, x_{n}^{\prime}=\left\{1+\frac{1}{n}\right\}, y_{n}^{\prime}=\left\{1-\frac{1}{n}\right\}, \\
& n \in N
\end{aligned}
$$

then the pairs $(A, S)$ and $(B, T)$ are weak compatible and satisfying $\operatorname{CLR}(g)$ So, all the conditions of our theorem are satisfied. Thus $A, B, S$ and $T$ have a unique common coupled fixed point in $X$. Indeed, $x=0$ is the unique common fixed point which is also a point of discontinuity and thus we find out an answer in fuzzy metric spaces to the problem of Rhoades[2].

\section{ApPlicAtion}

1) Theorem 3.2.

Let $(X, M, *)$ be a Fuzzy Metric Space, * being continuous t-norm with $a * b \geq a b, \forall a, b \in[0,1]$. Let $A, B: X \times X \rightarrow$ $X$ and $S, T: X \rightarrow X$ be four mappings satisfying following conditions:

1) The pairs $(A, S)$ and $(B, T)$ satisfy E.A property

2) $A(X \times X) \sqsubseteq T(X), B(X \times X) \sqsubseteq S(X)$,

3) $S(X)$ and $T(X)$ are closed subsets of $X$.

4) $M(A(x, y), B(u, v), k t) \geq \phi\{M(S x, T u, t) * M(A(x, y), S x, t) *$ $M(B(u, v), T u, t)\}$

$\forall x, y, u, v \in X, k \in(0,1)$ and $\phi:[0,1] \rightarrow[0,1]$ such that $\phi(t)>t$ for $0<t<1$.Then $(A, S)$ and $(B, T)$ have point of coincidence. Moreover if the pairs $(A, S)$ and $(B, T)$ are weakly compatible, then there exists unique $\mathrm{x}$ in $\mathrm{X}$ such that $A(x, x)=T(x)=B(x, x)=S(x)=x$. 


\section{REFERENCES}

[1] M. Aamri and D. El Moutawakil, "Some new common fixed point theorems under strict contractive conditions," Journal of Mathematical Analysis and Applications, vol. 27, pp. 181 - 188, 2002.

[2] B. E. Rhoades, "Contractive definitions continuity," Contemp. Math, vol. 72, pp. 233-245, 1988.

[3] T. G. Bhaskar and V. Lakshmikantham, "Fixed point theorems in partially ordered metric spaces and applications," Nonlinear Analysis: Theory, Methods and Applications, vol. 65, no. 7, pp. 1379-1393, 2006

[4] J. X. Fang, "Common fixed point theorems of compatible and weakly compatible maps in menger spaces," Nonlinear Analysis: Theory, Methods and Applications, vol. 71, no. 5-6, pp. 1833-1843, 2009.

[5] X.-Q. Hu, "Common coupled fixed point theorems for contractive mappings in fuzzy metric spaces," Fixed Point Theory and Applications, article id 363716, pp. 14, 2011

[6] V. Lakshmikantham and L. Ćirić, "Coupled fixed point theorems for nonlinear contractions in partially ordered metric spaces," Nonlinear Analysis: Theory, Methods and Applications, vol. 70, no. 12, pp. 4341-4349, 2009.

[7] S. Sedghi, I. Altun, and N. Shobe, "Coupled fixed point theorems for contractions in fuzzy metric spaces," Nonlinear Analysis: Theory, Methods and Applications, vol. 72, no. 3-4, pp. 1298-1304, 2010.
[8] W. Sintunavarat and P. Kuman, "Common fixed point theorems for a pair of weakly compatible mappings in Fuzzy Metric Spaces," Journal of Applied Mathematics, Article ID 637958, DOI: 10.1155/2011/637958, pp. 14, 2011

[9] L. A. Zadeh, "Fuzzy Sets," Information and Control, vol. 89, pp. 338-353, 1965.

[10] M. K. Mishra et.al, "Fixed point theorems in fuzzy metric space for weakly compatible maps satisfying integral type inequality," Int $J$. Applied Engineering Research, Dindigul, vol. 1, no. 3, 2010.

[11] M. Imdad and J. Ali, "Some common fixed point theorems in fuzzy metric space," Mathematical Communications vol. 11, pp. 153-163, 2006

[12] S. Sedhi and N. Shobe, "Fixed point theorems in M- Fuzzy Metric Spaces with property (E)," Advances in Fuzzy Mathematics ,ISSN 0973-533X,vol. 1, no.1, pp. 55-65, 2006

[13] V. Pant and R. P. Pant, "Fixed point theorems in Fuzzy Metric for non compatible maps," Soocho J. Mathematics, vol. 33, no. 4, pp. 647-665, October 2007.

[14] V. S. Chaun et.al, "Fixed point theorems in Fuzzy Metric for weakly compatible maps," Int. J. Contemp. Math Sci. vol. 5, no. 3, pp. 145-151, 2010 . 